ing, detailing not only the causes but also the consequences of change for both landscape and people. However, the influence of environmental change on some human activities, such as the origins of settled agriculture, is not made at all clear. The chapters that detail the effects of people on the environment provide useful synopses concentrating on erosion, which is examined on a regional basis, and climate. The chapter on conserving the cultural landscape falls, somewhat uncomfortably, between the topics of erosion and climate but its subject matter is both valuable and novel. Once again, the complex and inalienable components of nature and culture are apparent, although to the book's detriment there are no suggestions for possible frameworks in which to examine the relationships between people and the environment. The same is true of the all-too-brief postscript. This, for good or ill, manages to avoid discussion of the, albeit poorly-defined, concept of sustainable development.

Overall, the book presents a worthwhile synthesis of the history of people and the environment. It is a timely reminder that the character of this relationship is about to change: the question is whether this change will be by design or by default.

A. M. Mannion is in the Department of Geography, University of Reading, PO Box 227, Whiteknights, Reading RG6 $2 A B$, UK.

Key issues relating to debates on environmental change and policy are also examined in the new introductory text Environmental Issues in the 1990s edited by A. M. Mannion and S. R. Bowlby. Published by Wiley, $£ 14.95$ (pbk).

\title{
Degrees of uncertainty
}

\section{E. H. Mamdani}

Fuzzy Systems Theory and Its Applications. By Toshiro Terano, Kiyoji Asai and Michio Sugeno. Academic: 1992. Pp. 268. £33, \$49.95.

VARIABLES applicable to such areas as expert systems, knowledge engineering and artificial intelligence often cannot be specified with exact numerical values, as only vague information about them is available. Fuzzy logic is a method for handling such inexact information by attempting to quantify value judgements in such a way that they are amenable to processing by a computer. Often this amounts to creating a fuzzy (or 'fuzzied') version of the conventional theory; the method incorporates rules for manipulating fuzzy sets, which are sets of values corresponding to a fuzzy proposition. Fuzzy set theory has already had practical applications, for example in regression analysis, mathematical programming, diagnosis and systems control. This book provides a good overview of fuzzy set theory, with separate chapters devoted to the research results in each of these areas.

Fuzzy set theory was first introduced by L. A. Zadeh in 1965; the first book on the subject, A. Kaufman's An Introduction to the Theory of Fuzzy Subsets (Academic), was published ten years later. Since then, many other books have appeared in the field, but they have mostly dealt with only research results. Only H. J. Zimmerman's Fuzzy Set Theory and Its Applications (KluwerNijhoff, 1985) had any real appeal as a textbook. So it is pleasing now to have this new introduction, which, although primarily aimed at Japanese universities, can also be used elsewhere. The book was originally published in 1987 in Japanese. The English translation is excellent and very readable, although its Japanese origins are still plain to see.

Fuzzy set theory has been developed by workers all around the world, but the theory has never had wide appeal to mainstream researchers in Europe and the United States. Only Japanese (and perhaps also Chinese) workers have so far felt that the theory addresses an important need to represent vague information and deal with it scientifically. The authors of this new book, who are eminent teachers and research workers in the field, may not convince many Western researchers of the theory's value, although they do provide an insight into the kind of contributions that Japanese workers see it making to computer-based applications.

E. H. Mamdani is in the Department of Electronic Engineering, Queen Mary and Westfield College, Mile End Road, London E1 4NS, UK.

\section{Autumn Books}

Nature's next review supplement is Autumn Books, which is published on 26 November. The issue is due to feature, among others, Ed Regis on Arthur C. Clarke, Igor Aleksander on The Turing Option and Martin Gardner on spontaneous combustion.

\section{The Fontana History of Chemistry} William H Brock

A work of synthesis for the 1990 s

Essential reading for all students of Chemistry

\section{The Fontana History of the Environmental Sciences} Peter J Bowler

First comprehensive survey of all developments in the study of the earth's environment 